\title{
ZELDA: a 3D Image Segmentation and Parent-Child relation plugin for microscopy image analysis in napari
}

1 Rocco D’Antuono ${ }^{*}$, Giuseppina Pisignano

$2{ }^{1}$ Crick Advanced Light Microscopy STP, The Francis Crick Institute, London, United Kingdom

$3 \quad{ }^{2}$ Department of Biology and Biochemistry, University of Bath, Bath, United Kingdom

4 * Correspondence:

5 Corresponding Author

6 rocco.dantuono@crick.ac.uk

$7 \quad$ Keywords: image analysis $1,3 D_{2}$, ,egmentation $_{3}$, Parent-Child $_{4}$, napari $_{5}$, plugin $_{6}$, microscopy $_{7}$, 8 measurement 8 . (Min.5-Max. 8)

9 Number of words: 3007 . Number of figures: 8.

10 Abstract

Bioimage analysis workflows allow the measurement of sample properties such as fluorescence intensity and polarization, cell number, and vesicles distribution, but often require the integration of multiple software tools. Furthermore, it is increasingly appreciated that to overcome the limitations of the 2D-view-based image analysis approaches and to correctly understand and interpret biological processes, a 3D segmentation of microscopy data sets becomes imperative.

Despite the availability of numerous algorithms for the 2D and 3D segmentation, the latter still offers some challenges for the end-users, who often do not have either an extensive knowledge of the existing software or coding skills to link the output of multiple tools. While several commercial packages are available on the market, fewer are the open-source solutions able to execute a complete $3 \mathrm{D}$ analysis workflow.

Here we present ZELDA, a new napari plugin that easily integrates the cutting-edge solutions offered by python ecosystem, such as scikit-image for image segmentation, matplotlib for data visualization, and napari multi-dimensional image viewer for 3D rendering. This plugin aims to provide interactive and zero-scripting customizable workflows for cell segmentation, vesicles counting, parent-child relation between objects, signal quantification, and results presentation; all included in the same open-source napari viewer, and "few clicks away".

\section{$27 \quad 1 \quad$ Introduction}

Microscopy and image analysis significantly contribute to the advancement of research in life sciences. However, researchers operating microscopes have to deal with a number of experimental challenges often requiring different types of image analysis procedures. For example, the counting of protein structures, such as the ProMyelocytic Leukemia Nuclear Bodies (PML NB) found involved in chromatin remodeling, telomere biology, senescence or viral infections [Lallemand-Breitenbach, 2018], is achievable by applying a " $2 \mathrm{D}$ counting" image analysis tool to first identify cells and then determine the number of contained PML NB (Suppl. Fig. 1A). Similarly, in experiments where the measurement of transient concentration 
of $\mathrm{Ca} 2+$ or metabolites is assessed, a stable staining and reliable segmentation of individual cytoplasmic organelles might be required to then apply a "2D measurement" of fluorescence intensity and organelle shape (Suppl. Fig. 1B). This can be fundamental in studies of mitochondrial metabolism where a complex correlation between ER-mitochondria Ca2+ fluxes and autophagy have been highlighted [Missiroli, 2020]. Furthermore, some kidney pathological conditions, such as the glomerulocystic disease, could originate from topological defects acquired during development [Fiorentino, 2020]. Such conditions can be studied using a staining to identify single cells, glomeruli, and the renal tubular system (Suppl. Fig. 1C). The conformational study of a glomerulus, with the assessment of the number of cells, is referred to as "3D cell counting" or "3D object segmentation". In influenza infection, instead, the released viral genome can be involved in mechanisms such as replication or viral protein transcription and identified by the presence of a negative-sense RNA [Long, 2019]. The dynamics of the viral infection can therefore be monitored by localizing the RNA molecules within the cell nuclei (Suppl. Fig. 1D) in a task definable as "3D object segmentation" and "parent-child relation".

The ability to extrapolate valuable results from microscopy experiments as those just mentioned, mainly relies on the image analysis knowledge and availability of the right software tools for the specific purpose. The bioimage analysis is a combination of multiple informatics tools (referred to as "components") organized into "workflows" with different levels of complexity [Miura, 2020]. Such components are often available only by scripting and researchers may struggle to find an effective way of combining them together in a complete workflow. To date, there have been great initiatives to both promote the bioimage analysis (NEUBIAS Training Schools [Martins, 2021]) and raise awareness about informatics tools (BioImage Informatics Index, http://biii.eu/), while a growing number of excellent open-source software became available [Schindelin, 2012] [McQuin, 2018]. However, the end-user has still to acquire a minimum level of bioinformatic knowledge in order to analyze image data.

A recent survey proposed by the $\mathrm{COBA}^{1}$ to the bioimage analysis community has suggested that the most used bioimage analysis tools belong to the category of the "open-source point and click software" and there is a high demand for better software for "3D/Volume" and "Tissue/Histology" analysis [Jamali, 2021], underlining the urgency of more and more new, easy and customizable tools for multi-dimensional image segmentation.

Furthermore, to guarantee the experimental reproducibility, minimize the mistakes, and preserve scientific integrity, any new analysis software should include accurate logging of the used parameters at each step of the workflow ${ }^{2}$.

To facilitate life science researchers during the application of image analysis to biological experiments, we developed ZELDA: a napari plugin for the analysis of 3D datasets with multiple object populations. ZELDA has the advantage of being equipped with ready-to-use protocols for 3D segmentation, measurement, and "parent-child" relation between object classes. It then allows the rapid cell counting, quantification of vesicle distribution, and the fluorescence measurement of subcellular compartments for most biological applications. Since

\footnotetext{
${ }^{1}$ Center for Open Bioimage Analysis: https://openbioimageanalysis.org/

${ }^{2}$ Kota Miura 2020, “In Defense of Image Data \& Analysis Integrity” - [NEUBIASAcademy@ Home] Webinar https://www.youtube.com/watch?v=c_Oi2HKom_Y
} 
each image analysis workflow is designed as a simple protocol with numbered steps, it requires no knowledge of image analysis and it's sufficient to follow the step-by-step instructions to perform a complete analysis. Furthermore, while the integration in napari allows to easily view each step of the image processing as 2D slice or 3D rendering, the visibility, opacity and blending modulation facilitates the tuning of the used parameters (for example threshold value or gaussian filter size) by visualizing multiple layers at the same time.

Altogether ZELDA plugin is a new easy to use open-source software designed to assist researchers in the most common bioimage analysis applications without requiring any scripting knowledge.

\section{Materials and Methods}

\subsection{Image acquisition}

The data sets of influenza infected human eHAP cells, BPAE cells (Invitrogen FluoCells Slide \#1) and mouse kidney tissue (Invitrogen FluoCells Slide \#1), shown and analyzed in (Fig. 2, Fig. 4, Fig.5, Suppl. Fig. 1, and Suppl. Fig. 3) have been acquired with a Zeiss LSM880 confocal microscope, using a Plan-Apochromat 20X/0.8 NA objective. A sequential acquisition for DAPI (excitation $405 \mathrm{~nm}$, detection in the range 420nm - $462 \mathrm{~nm}$ ), AlexaFluor 488 and AlexaFluor 568 (excitation $561 \mathrm{~nm}$, detection in the range 570nm - $615 \mathrm{~nm}$ ) was used to acquire z-stacks with the total size up to $13 \mathrm{um}$, every $0.5 \mathrm{um}$. Pixel size was $0.20 \mathrm{um}$.

The beads used to show the segmentation workflow (Fig. 1) were TetraSpeck ${ }^{\mathrm{TM}}$ Microspheres, $0.1 \mu \mathrm{m}$; images were acquired on a Zeiss Observer.Z1 using Micro-Manager (https://micromanager.org/) software with a Hamamatsu ORCA-spark Digital CMOS camera, using a $63 \mathrm{X} / 1.4$ NA objective. Pixel size is 0.08 um.

\subsection{Object segmentation, measurements, and results export}

The segmentation obtained by running the ZELDA protocols is achieved using scikit-image [van der Walt, 2014] (version 0.18.1) and SciPy [Virtanen, 2020] (version 1.6.3) modules for image processing in python.

The resulting measurements are handled as Pandas data frames [McKinney, 2011] (version 1.2.4) and plotted with Matplotlib [Hunter, 2007] (version 3.4.2).

\subsection{Graphical User Interface (GUI) design, plugin development, installation, and execution}

ZELDA plugin for napari ("napari-zelda") can be installed through the "Install/Uninstall Package(s)" menu in napari [napari contributors, 2019], and its interface can be added with "Plugins/Add dock widget".

Alternatively, the installation can be done downloading the repository, navigating to it with the Anaconda prompt and using the command "pip install -e ." within the downloaded folder.

The plugin widgets have been created using magicgui [https://github.com/napari/magicgui], while the GUI plots included in the "Data Plotter" protocol are obtained with matplotlib.backends.backend_qt5agg [https://matplotlib.org/2.2.2/_modules/matplotlib/backends/backend_qt5agg.html]. 


\subsection{JSON database for modularity of the GUI and customization of image analysis protocols}

Once the user has selected a specific base protocol, a JSON file is used by the plugin to load the right widgets in the GUI.

The "Design a new Protocol" option saves the custom workflow as a list of widgets that will be sequentially loaded the next time that the newly created protocol is launched. It will be visible just after relaunching napari and ZELDA plugin.

3.1 ZELDA Protocols as an easy way to run image analysis workflows for $2 D$ and 3D segmentation

ZELDA plugin for napari ("napari-zelda") makes available to the end-user the segmentation, measurement, and "parent-to-child" relation of two object populations. It ultimately allows to plot the results and explore the data in the same Graphical User Interface (GUI).

The current version of the plugin includes three different "protocols" to ease the image analysis of 3D datasets. Each protocol is a set of individual steps (functions) that return images (as napari layers), or results (printed plots in .tiff or tables in .csv format).

The first protocol, called "Segment a single population" (Fig. 1A), can be used to segment both the 2D or 3D data sets. The basic workflow of this protocol (Fig. 1B) includes simple steps, such as Gaussian Blur, Threshold, and Distance Map, to identify the seed points for the subsequent segmentation of the objects of interest. The user can then set the "min dist" parameter in the "Show seeds" function to improve the accuracy of cell counting, before calling the "Watershed" segmentation (Fig. 1C-H). The detected objects can eventually be measured and the results table automatically saved (Suppl. Fig. 2A).

Similar workflows have been previously implemented in useful tools such as MorphoLibJ [Legland, 2016] and in the latest versions of CellProfiler [McQuin, 2018], although with the limitation of being exclusively applied to the 2D image analysis, or lacking an embedded and flexible 3D viewer. In contrast, ZELDA provides an integration of a basic 3D object segmentation workflow with napari 3D rendering GUI. Notably, in ZELDA the individual workflow steps are also accessible as single functions that can be optionally used, or fine-tuned individually, without having to restart the entire workflow from scratch.

The second protocol, "Segment two populations and relate" (Fig. 2A), implements the segmentation of two populations of objects in parallel, using the same workflow described above, with an additional step that allows establishing the "parent-child" relation between the two object populations (Fig. 2B-D).

To run reproducible image analysis with ZELDA, both described protocols include a "log" functionality that stores the parameters used at each step. The log is shown in the GUI and can be optionally saved as a .txt file, together with the other results (Suppl. Fig. 2B). 
Once segmented, measured, and optionally related two object populations, the "Data Plotter" protocol (Fig. 2E) allows to load a result table, and plot histograms or scatterplots of the measured properties. The plots are shown directly in the napari GUI and can be automatically saved as images to a specific folder. This has the advantage of avoiding the employment of additional software for data visualization.

Given that ZELDA does not require any coding skill, life science researchers are hugely facilitated by the integration of multiple bioinformatics tools in a single GUI.

\subsection{Modularity of the ZELDA Graphical User Interface allows to easily customize bioimage analysis workflows without any scripting knowledge}

Computer scientists and developers continuously propose new algorithms to tackle biological problems that frequently require extensive coding skills. However, users might have the necessity to reproduce a specific published workflow (such as the one in Fig. 1B), without knowing a scripting language or necessarily having any background in image analysis. We made this possible by implementing a method that allows the customization of the image analysis protocols available in ZELDA. Indeed, by simply running the fourth option called "Design a New Protocol", a user can easily create a new custom protocol (Fig. 3A). Every step of the base protocols is listed in a JSON database and the relative GUI widgets (used for the software layout) are available as ready-to-use modules to build personalized protocols. The different functions, such as threshold, gaussian blur or distance map etc., can be chosen in a drop-down menu at specific steps of the new protocol (Fig. 3B). By using the saving option (Fig. 3C), the JSON database will be automatically updated (Fig. 3D), and the ordered series of GUI widgets will be available the next time that ZELDA plugin will be launched (Fig. 3E).

\subsection{ZELDA segmentation and parent-child relation have the same accuracy of ImageJ and CellProfiler in 2D and 3D data sets, and the execution is twice faster}

In order to assess the accuracy in the segmentation of 2D and 3D data sets, we compared the results obtained by the ZELDA plugin for napari with those generated by two of the most widely used software in the bioimage field: ImageJ and CellProfiler.

As 2D data sets, we used images of cells (Fig. 4A) at a low confluence ( $30 \%$ of the field of view area) with a cytoplasmic staining to identify parent objects, and a second one for cellular organelles (children objects), with the final goal of correctly assign the organelles to the containing cell (parent-child relation).

Intriguingly, ZELDA performed almost equivalently to ImageJ (Fig. 4B) in identifying parent objects (Jaccard index $\mathrm{J}=0.987+/-0.002)$, child objects $(\mathrm{J}=0.920+/-0.031)$, and in the parentchild relation $(\mathrm{J}=0.993+/-0.001)$. This means that, assuming ImageJ segmentation as ground truth (Fig. 4G-I), ZELDA will correctly label the pixels of an organelle as belonging to the corresponding cell cytoplasm in $99 \%$ of the cases (Fig. 4D-F).

However, the adherence with CellProfiler labelling was slightly less striking (Fig. 4C) although this difference might be due to the many more parameters available in the CellProfiler GUI, such as the "declump method" in the "watershed" module et c., that have not been implemented in ZELDA GUI to keep the software interface and its utilization as simple as possible. Nonetheless, the agreement on the identification of the parent cytoplasms found with 
CellProfiler (Fig. 4J) was around $88 \%$ of the pixels, while for both the child objects segmentation and the parent-child relation (Fig. 4K-L) it was $\sim 82 \%$.

Benchmarking the segmentation of 3D data sets has proven to be slightly more complicated, since not all the available modules in CellProfiler support the 3D data processing. For example, in version 4.2.1 the "smooth" module that operates a Gaussian blur filter, is available just for the 2D data pipeline, while another one has to be used for the 3D case. The same holds for morphological operations such as those executed by the "ExpandOrShrinkObjects". Trying to circumvent this lack of interchangeable 2D/3D functions could result in a more elaborated and time-consuming construction of the CellProfiler pipeline. Conversely, the versatile protocols supplied with ZELDA (Fig. 1 and Fig. 2A-D) allowed the 3D segmentation and parent-child relation in fewer steps and about twice quicker than the CellProfiler "Test mode" (Fig. 5J).

We then analyzed a collection of z-stacks of mouse kidney glomeruli, as 3D data sets (Fig. 5AC). In this tissue, phalloidin staining (Fig. 5B) was used for the identification of the glomerular structures, and DAPI staining (Fig. 5A) to pinpoint the cell nuclei contained in each glomerulus. The resulting segmentation of the two populations and parent-child relation obtained by ZELDA (Fig. 5D-F) were compared with the output of a CellProfiler pipeline which included solely the 3D data compatible modules (Fig. 5G-I).

Unfortunately, the labelling agreement between the two software was reduced with respect to the $2 \mathrm{D}$ analysis. A performance comparison of the 3D segmentation revealed a variation of the Jaccard index across the z-stack, with maximum values typically around the mid-slice, where the staining intensity of the confocal microscopy data set was stronger (Suppl. Fig. 3A). We then considered the maxima of the Jaccard index across the Z-stacks (Fig. 5K), assessing an accordance around $63 \%$ for the parent objects (Jaccard index $\mathrm{J}=0.632+/-0.011$ ), $73 \%$ for the children $(\mathrm{J}=0.735+/-0.011)$, and of $64 \%$ for the parent-child relation $(\mathrm{J}=0.643+/-0.010)$

(Suppl. Fig. 3B).

We further investigated the reason for the lack of agreement on 3D data sets labelling between ZELDA and CellProfiler, and found that the difference was due to the absence of 3D equivalents for some modules (e.g. the "ExpandOrShrink" morphological operations), or lack of a unique naming for the 2D and 3D version of the same method in CellProfiler (e.g. "Gaussian Blur"). Indeed, pre-processing the z-stacks with the ZELDA and proposing the resulting smoothed 3D data sets to CellProfiler, successfully increased the accordance in identifying parents, children, and parent-child relation above the $99 \%$ of the pixels (Fig. 5L).

Therefore, ZELDA can represent a faster interactive alternative to CellProfiler for the exploratory analysis of 3D data sets.

Many tools are available for 2D segmentation, while fewer are able to process 3D data sets [Schindelin, 2012] [McQuin, 2018] [Berg, 2019]. The main limitation is frequently due to the lack of a flexible 3D viewer to render the resulting processed images (segmented 
a complete 3D analysis workflow may demand different levels of background knowledge in coding and image analysis.

Considering the growing request for bioimage analysis tools and the difficulties encountered by the users, we developed ZELDA, a plugin for 3D image segmentation and parent-child relation for microscopy image analysis in napari [napari contributors, 2019].

ZELDA plugin has the flexibility of being applicable to different purposes and data sets, such as the image measurement of beads to assess microscope resolution (Fig. 1B), the RNA quantification in influenza-infected human cell nuclei (Fig. 2B-D), the identification of cellular compartments and organelle counting in cell culture samples (Fig. 4D-F), or the morphological characterization of organs and tissues (Fig. 5D-F).

The 2D and 3D image analysis workflows that ZELDA protocols convey (Fig. 1A and Fig. 2A) do not require an extensive knowledge of the used algorithms, coding skills, or an elevated number of "point and click" interactions.

The "Data Plotter" protocol (Fig. 2E) enables the data exploration during the image analysis, favoring the biological sample comprehension, and potentially highlighting differences between treatments "on the fly". Furthermore, the reproducibility of workflows is sustained by the implementation of the $\log$ (Suppl. Fig. 2B) and persistence in memory of the previously used image analysis parameters (i.e. restarting the same protocol will show the parameters values used during the last run).

The implementation of image analysis workflows found in literature is achievable with a fourth protocol called "Design a New Protocol" (Fig. 3 A-C). Without any scripting, users can manage the available "widgets" to create a custom GUI (Fig. 3 E) that can then be saved and shared with the community (by sharing the JSON database) (Fig. 3 D).

Nonetheless, through the customization of the GUI allowed by the fourth protocol, a simply different use of the already available functionalities can lead to better object segmentation. For example, including an additional "Threshold" step after the "Get DistanceMap", in a newly designed protocol, could help to remove smaller debris before "Show seeds". Certainly, the possibility of rearranging the components of the image analysis workflows, by using an immediate graphical mode, represents a valuable contribution as an open-source software to bioimage analysis.

To date, ZELDA presents a minimalist interface with three basic protocols implementing image analysis workflows but it could be easily be powered up with additional processing steps to improve image segmentation (e.g. morphological operators to moderate under and oversegmentation, a filter module to exclude segmented objects by intensity or shape descriptors, or allowing to deconvolve the data set before segmenting it).

Although still unable to process images in batch mode, ZELDA can find its niche of comparable level of ImageJ and CellProfiler in 2D. While in 3D, the segmentation and "parent-child" relation of multi-class objects is performed with a shorter implementation of the workflows and twice faster. 
In conclusion, ZELDA plugin for napari can hugely accelerate and facilitate the applications of

\section{Acknowledgements}

We thank Sara Barozzi (IEO, Milan) for the preparation of the PML NB sample shown in Suppl. Fig. 1A.

We thank Olivia Swann (Barclay Lab, Imperial College London) for supplying the influenza infected cells analyzed in Suppl. Fig. 1D.

\section{Conflict of Interest}

The authors declare that the research was conducted in the absence of any commercial or financial relationships that could be construed as a potential conflict of interest.

\section{$7 \quad$ Authors contribution}

RDA developed the napari-zelda plugin, acquired and analyzed the data, and wrote the manuscript. GP helped to develop the plugin and wrote the manuscript.

RD'A works at the Francis Crick Institute which receives its core funding from Cancer Research UK (FC001999), the UK Medical Research Council (FC001999), and the Wellcome Trust (FC001999).

9 Data Availability Statement

The datasets [GENERATED/ANALYZED] for this study can be found in the https://github.com/RoccoDAnt/napari-zelda .

\section{References}

Berg, S., Kutra, D., Kroeger, T. et al. ilastik: interactive machine learning for (bio)image analysis. Nat Methods 16, 1226-1232 (2019). https://doi.org/10.1038/s41592-019-0582-9

302 John D. Hunter. Matplotlib: A 2D Graphics Environment, Computing in Science \& Engineering, 9, 303 90-95 (2007), DOI:10.1109/MCSE.2007.55

304 Nasim Jamali, Ellen TA Dobson, Kevin W. Eliceiri, Anne E. Carpenter, Beth A. Cimini, 2020

305 BioImage Analysis Survey: Community experiences and needs for the future, bioRxiv

306 2021.08.16.456498; doi: https://doi.org/10.1101/2021.08.16.456498 
307 Lallemand-Breitenbach V, de Thé H. PML nuclear bodies: from architecture to function. Curr Opin

308 Cell Biol. 2018 Jun;52:154-161. doi: 10.1016/j.ceb.2018.03.011. Epub 2018 Apr 30. PMID:

30929723661.

310 David Legland, Ignacio Arganda-Carreras, Philippe Andrey, MorphoLibJ: integrated library and 311 plugins for mathematical morphology with ImageJ, Bioinformatics, Volume 32, Issue 22, 15

312 November 2016, Pages 3532-3534, https://doi.org/10.1093/bioinformatics/btw413

313 Long, J.S., Mistry, B., Haslam, S.M. et al. Host and viral determinants of influenza A virus species

314 specificity. Nat Rev Microbiol 17, 67-81 (2019). https://doi.org/10.1038/s41579-018-0115-z

315 Martins GG, Cordelières FP, Colombelli J et al., Highlights from the 2016-2020 NEUBIAS training 316 schools for Bioimage Analysts: a success story and key asset for analysts and life scientists [version

317 1; peer review: 2 approved]. F1000Research 2021, 10:334

318 (https://doi.org/10.12688/f1000research.25485.1)

319 McKinney, Wes. "pandas: a Foundational Python Library for Data Analysis and Statistics.” (2011).

320 McQuin C, Goodman A, Chernyshev V, Kamentsky L, Cimini BA, Karhohs KW, Doan M, Ding L, 321 Rafelski SM, Thirstrup D, Wiegraebe W, Singh S, Becker T, Caicedo JC, Carpenter AE (2018).

322 CellProfiler 3.0: Next-generation image processing for biology. PLoS Biol. 16(7):e2005970 / doi.

323 PMID: 29969450 (Research article)

324 Missiroli S, Perrone M, Genovese I, Pinton P, Giorgi C. Cancer metabolism and mitochondria:

325 Finding novel mechanisms to fight tumours. EBioMedicine. 2020 Sep;59:102943. doi:

326 10.1016/j.ebiom.2020.102943. Epub 2020 Aug 17. PMID: 32818805; PMCID: PMC7452656.

327 Miura K, Paul-Gilloteaux P, Tosi S, Colombelli J. Workflows and components of bioimage analysis.

328 In: Miura K, Sladoje N, editors. Bioimage Data Analysis Workflows. Learning Materials in

329 Biosciences. Cham: Springer (2020)

330 napari contributors (2019). napari: a multi-dimensional image viewer for python.

331 doi:10.5281/zenodo.3555620

332 Schindelin, J., Arganda-Carreras, I., Frise, E. et al. Fiji: an open-source platform for biological-image 333 analysis. Nat Methods 9, 676-682 (2012). https://doi.org/10.1038/nmeth.2019

334 van der Walt S, Schönberger JL, Nunez-Iglesias J, Boulogne F, Warner JD, Yager N, Gouillart E, Yu 335 T, the scikit-image contributors. 2014. scikit-image: image processing in Python. PeerJ 2:e453

336 https://doi.org/10.7717/peerj.453

337 Virtanen P, Gommers R, Oliphant TE, Haberland M, Reddy T, Cournapeau D, Burovski E, Peterson 338 P, Weckesser W, Bright J, van der Walt SJ, Brett M, Wilson J, Millman KJ, Mayorov N, Nelson 339 ARJ, Jones E, Kern R, Larson E, Carey CJ, Polat İ, Feng Y, Moore EW, VanderPlas J, Laxalde D, 340 Perktold J, Cimrman R, Henriksen I, Quintero EA, Harris CR, Archibald AM, Ribeiro AH, 341 Pedregosa F, van Mulbregt P; SciPy 1.0 Contributors. SciPy 1.0: fundamental algorithms for 342 scientific computing in Python. Nat Methods. 2020 Mar;17(3):261-272. doi: 10.1038/s41592-019343 0686-2. Epub 2020 Feb 3. Erratum in: Nat Methods. 2020 Feb 24;: PMID: 32015543; PMCID:

344 PMC7056644. 
346 Figure 1. ZELDA plugin for napari. (A) GUI of ZELDA with the description of the ready-to-use protocols sufficient to run a complete image analysis workflow (white text box on the top right). Each protocol is divided into numbered steps corresponding to the software commands in the napari dock widget (bottom of the software interface). (B) "Segment a single population" protocol including a minimum number of processing operations. (C) Original image. (D) Gaussian Blur of the original image. (E) Binary image obtained applying a Threshold to the gaussian blur. (F) Distance Map applied to the binary image. (G) Seeds (Local Maxima) used to run the Watershed. (H) Objects labelled by Watershed. The labelled objects can then be measured, and the results exported.

Figure 2. ZELDA application for the 3D segmentation of two object populations and "parentchild" relation. (A) ZELDA protocol "Segment two populations and relate" used to analyze the distribution of viral RNA in infected human cell nuclei. (B) Original 3D data set showing a nuclear staining with DAPI (gray) and an RNA staining with AlexaFluor 568 (red). (C) The nuclei and the RNA aggregates, individually segmented and (D) the RNA aggregates (children population) labelled according to the containing nuclei (parent population). (E) Resulting measurements reimported and plotted with the "Data Plotter" protocol.

Figure 3. Design of a custom image analysis workflow with ZELDA without requiring any image analysis workflow. (B) Drop-down menu showing all the modules implemented in ZELDA. (C) Assignment of an operation to a specific step of the new protocol. (D) Example of updated JSON database that controls the software layout, once a new protocol is saved. (E) The newly created protocol GUI available after having restarted ZELDA. CellProfiler. (A) 2D images of BPAE cells stained with DAPI (blue, cell nuclei), AlexaFluor 488 (green, cytoplasms), and MitoTracker Red (red, mitochondria). (B) Labelling comparison between ZELDA and ImageJ showing an accordance above the $98 \%$ of the pixels for "parent" objects (cell cytoplasms), 92\% for "child" objects (mitochondria), and 99\% for the parent-child relation. (C) Comparison between ZELDA and CellProfiler showing a minor accordance still above the $88 \%$ of the pixels for "parents", $82 \%$ for "children", and $82 \%$ for the "parent-child" relation. ZELDA labelling of (D) cell cytoplasms, (E) mitochondria, and (F) masked mitochondria (parent-child relation). ImageJ labelling of $(\mathbf{G})$ cell cytoplasms, $(\mathbf{H})$ mitochondria, and (I) masked mitochondria (parent-child relation). CellProfiler labelling of (J) Cell cytoplasms, $(\mathbf{K})$ mitochondria, and $(\mathbf{L})$ masked mitochondria (parent-child relation).

Figure 5. 3D segmentation, "parent-child" relation, and execution time of ZELDA compared to CellProfiler. Z-stacks of mouse kidney tissue showing glomeruli were used for the benchmark in 3D. (A) DAPI staining used to segment the cell nuclei. (B) Phalloidin used to identify the glomerular structures. (C) 3D rendering showing the merge of DAPI (gray), WGA (green), and phalloidin (red). (D) Nuclei, (E) glomerular structures, and (F) masked nuclei (parent-child relation) labelled by ZELDA in 3D. (G) Nuclei, (H) glomerular structures, and (I) masked nuclei (parent-child relation) labelled by CellProfiler in 3D (using a pipeline containing only 3D data compatible modules). (J) Execution time for the same workflow developed as both CellProfiler pipeline and ZELDA protocol, with the goal to segment in 3D and relate parents and children objects. The boxplots represent the distribution of multiple runs analyzing individual FOVs. For CellProfiler in batch mode, the CPU time has been considered, while the blue dot represents the total duration 
389 experienced by the end user for the analysis of 9 FOVs (including the wall time). (K) Variation of the 390 Jaccard index of the segmentation obtained with ZELDA and CellProfiler, around the mid-slice

391 where the signal is stronger. In the 3D case, the maxima of the Jaccard scores along the Z-stack were

392 used for the benchmarking. Not all the CellProfiler modules are 3D compatible, then the execution of

393 a minimal pipeline may result in over-segmented structures. The reason was identified to be the lack

394 of a unique name for the same operation in 2D and 3D ("Smooth"), or the absence of 3D equivalents

395 for some modules like the "ExpandOrShrink" morphological operations. CellProfiler might be able

396 to process the data sets equivalently to ZELDA but with a longer and more complicated pipeline. (L)

397 Increment of agreement on segmentation above the $99 \%$ once a pre-processed 3D data by ZELDA

398 was proposed to CellProfiler, showing how quickly ZELDA can segment and relate in 3D using less

399 steps than a CellProfiler pipeline. 
Fig 1
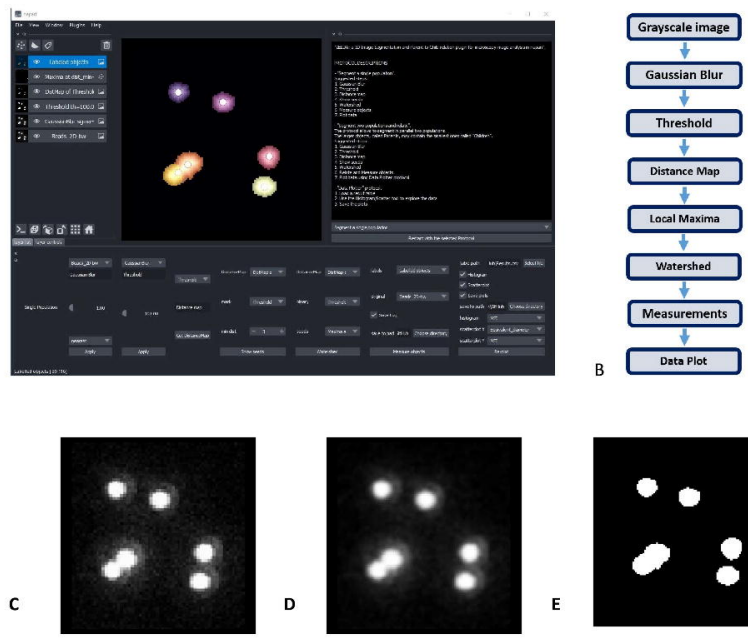

D
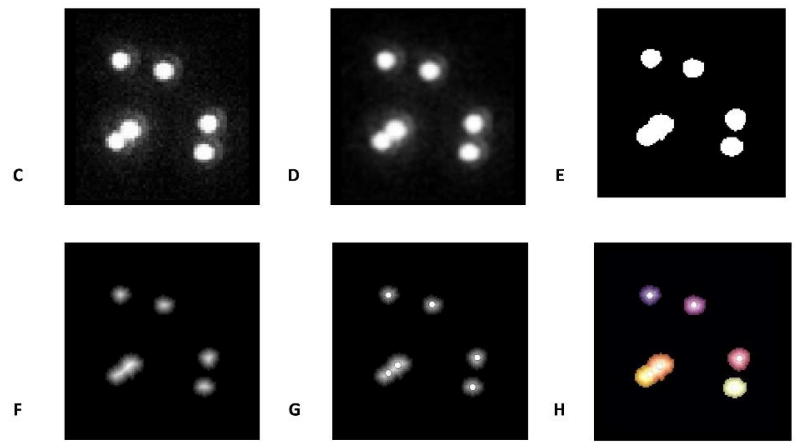
Fig 2

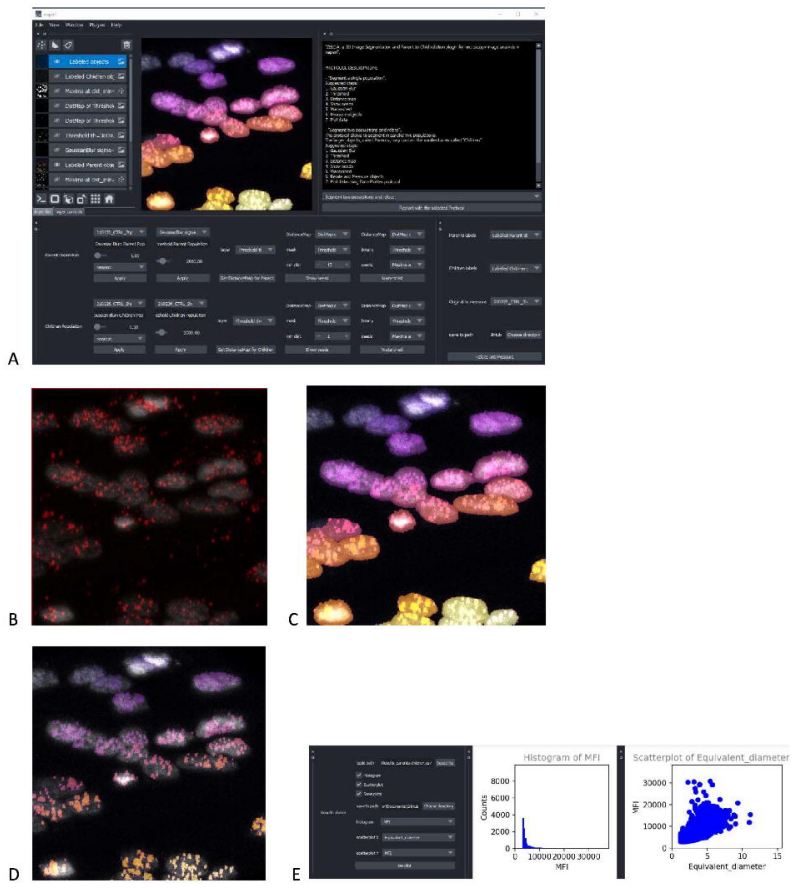


Fig 3

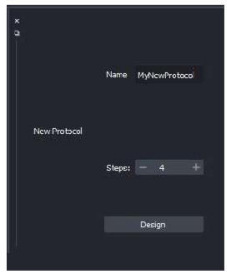

Nane MWNewProtcco

A

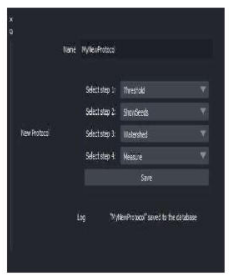

C

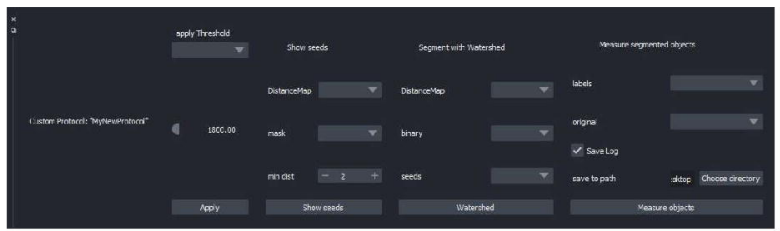

E
B

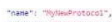

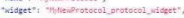

"3tess"t 1

step_punter"it: $t$.

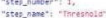

1.

intep_nimber": 2,

-step_nase": -shisuseds

7.

miten nusser":

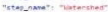

i.

atep_nunterni, 4,

istep nare"t measure"

D

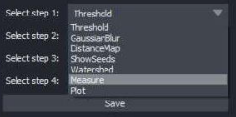


Fig 4

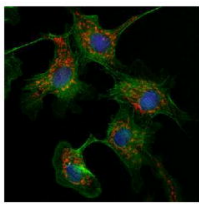

A

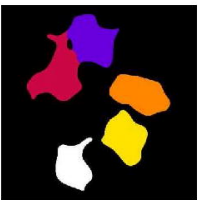

D

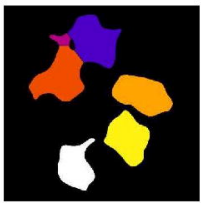

G

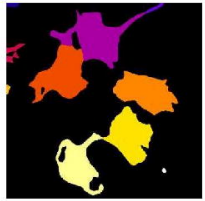

J

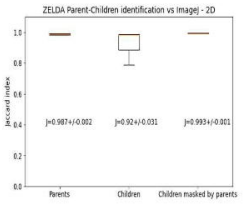

B

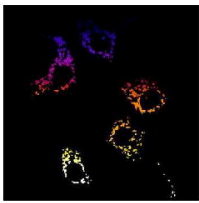

E

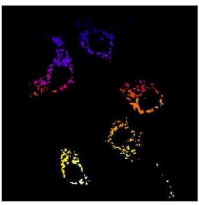

H

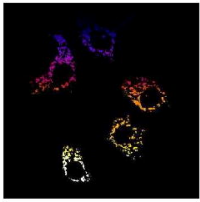

K

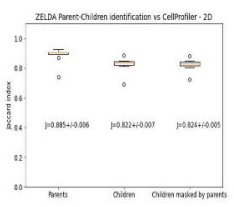

C

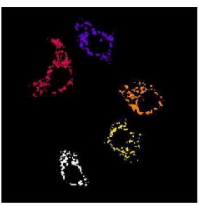

F
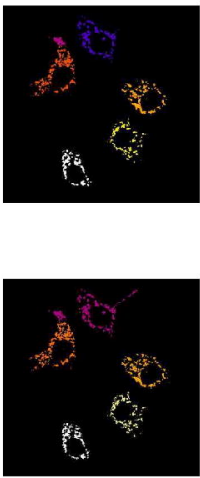

L 
Fig 5

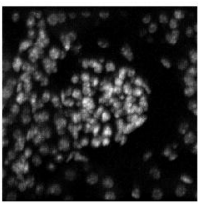

A

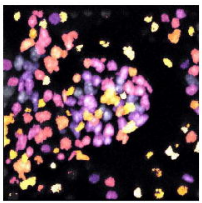

D

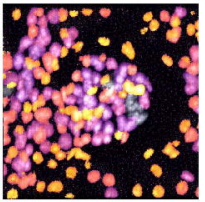

G

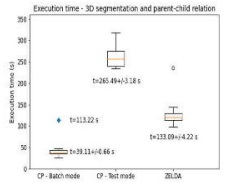

J

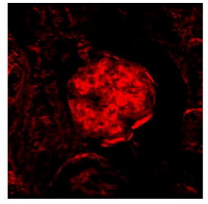

B

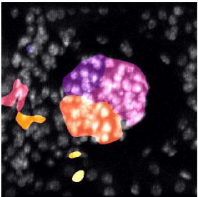

E

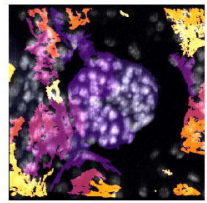

H

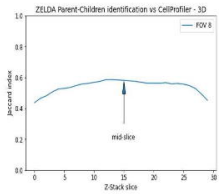

K

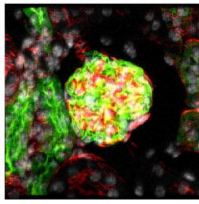

C

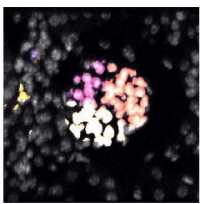

F
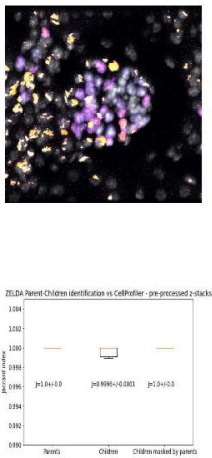

L 